\title{
Gastos públicos e crescimento econômico: evidências da economia do estado do Ceará
}

\author{
Public spending and economic growth: evidences \\ from the economy of the state of Ceará
}

\author{
Jair Andrade de Araujo ${ }^{1}$ \\ Vitor Borges Monteiro ${ }^{2}$ \\ Gabriel Alves de Sampaio Morais ${ }^{3}$
}

\section{Resumo}

O artigo tem o intuito de explanar a relação entre os gastos públicos com o crescimento econômico, trazendo uma revisão da literatura e uma análise da influência de algumas despesas sobre o crescimento econômico nos municípios cearenses. As despesas são com: saúde e saneamento, urbanismo e habitação, custeio de pessoal e legislativo. O capital físico e humano dos municípios cearenses são incorporados. Para essa análise, utiliza-se uma amostra de 122 dos 184 municípios cearenses, no período de 2002 a 2009, em uma abordagem dinâmica de acordo com Arellano e Bond (1991). Como modelo teórico, adotou-se uma extensão da função de produção de Barro (1990), cujo objetivo é justamente tentar encontrar, para os municípios cearenses, quais tipos de gastos estariam influenciando seu crescimento econômico. Conclui-se que os incrementos em capital físico e humano têm contribuído para elevar o crescimento nos municípios cearenses. Ressalta-se que o impacto do capital humano é maior do que o obtido via crescimento do capital físico. Dessa forma, políticas públicas direcionadas ao aumento da educação apresentam maiores impactos no aumento do produto do que aquelas que influenciam apenas o crescimento do produto com elevação do capital físico. Os gastos com saúde, saneamento e legislativos não apresentam relação com o produto. Já as despesas com urbanismo, habitação e custeio com pessoal apresentaram impacto positivo sobre o PIB per capita.

Palavras-chave: Finanças Públicas. Gastos Públicos. Crescimento Econômico.

1 Doutor em Economia. Profo . Adjunto dos Cursos de Economia e Finanças da Universidade Federal do Ceará/Campus de Sobral. Rua Anahid Andrade, s/n - Centro e CEP: 62.011-000. Sobral, CE - Brasil. E-mail: jairandrade@ufc.br

2 Doutor em Economia. Prof ${ }^{\circ}$. Adjunto dos Cursos de Economia e Finanças da Universidade Federal do Ceará/Campus de Sobral. Rua Anahid Andrade, s/n - Centro e CEP: 62.011-000 Sobral, CE - Brasil. E-mail: vimonteiro5@hotmail.com.br

3 Graduado em Análise e Desenvolvimento de Sistemas. Rua Dr. Floro Bartolomeu, 862 - Centro. Juazeiro do Norte, CE - Brasil Email: gabriel_morais@yahoo.com.br. 


\section{Abstract}

This paper seeks to shed light on the relationship of public spending and economic growth. Its main concern is guided by two tasks: an executive summary, e.g, a review literature but also to develop a view of the role played by some real spending within cearenses municipalities and its economic growth. The expenses consider: public health and sanitation; urbanism and dwelling, employee's payroll and legislature. The physical and human capital is added into the explanation. For, one makes use of an equivalent amount to 122 from 184 cearenses municipalities regarding the 2002-2009 period. It assumes a dynamic approach in Arellano-Bond (1991) outlook. The problematic will be discussed within the framework of Barro idiosyncratic tenet: his so-called 1990 production function which has precisely as main aim to discover which kind of spendings would be influencing the economic growth of cearenses municipalities as well. All in all the following points are reached: physical and human capital is decisive for increasing economic growth of cearenses municipalities. Regardless to say that human capital is better placed than the physical one. As an immediate consequence, public policies should be required especially the ones concern education which are really urgent. Health and sanitation expenses as well legislature have no relationship to outcome. However, the expenses related to urbanism and dwelling, employee's payroll had positive impact on GDP per capita.

Keywords: Public Finance. Public Spending. Economic Growth.

\section{Introdução}

Gasto público é um dos temas mais discutidos no âmbito das finanças públicas. É também um dos principais alvos das críticas dos economistas, sobretudo os que defendem maior eficiência. Ter conhecimento de como melhor alocar os recursos do governo é de grande utilidade à toda sociedade, e para isso toma-se o Produto Interno Bruto (PIB) de uma economia como forma de acompanhar a eficiência dos gastos governamentais.

Há muitos anos, estudiosos analisam os impactos dos gastos públicos sobre o crescimento econômico. Uma das linhas de argumentação é a de que sucessivas elevações desses gastos tenderiam a limitar a formação de poupança interna e os incrementos dos investimentos privados, além da consequente diminuição do produto. Esses argumentos foram construídos a partir de modelos tradicionais de 
crescimento, a saber, o de Solow (1956): o efeito final de elevações de gastos seria a redução dos níveis do produto per capita.

Por outro lado, muitas questões foram levantadas a partir do modelo de crescimento endógeno de Barro (1990), que demonstra a relação entre os gastos públicos e o crescimento. O impacto dos gastos governamentais é nulo, desde que não afete a produtividade do setor privado.

A partir de então, surgem diversos estudos que apregoam a divisão dos gastos públicos em dois tipos: aqueles considerados improdutivos, pois não afetam o crescimento de longo prazo; e os produtivos, que, introduzidos na função de produção local, relacionam-se positivamente com o crescimento econômico de longo prazo (SILVA, 2012).

Por exemplo, Aschauer (1989), ao analisar a relação dos gastos públicos com o crescimento econômico, conclui que eles provocam elevações no produto. Ele utilizou dados da economia dos EUA e afirmou ser possível um aumento de 0,36 a 0,39 no produto, com o aumento de $1 \%$ nos gastos em infraestrutura.

Na mesma perspectiva, tem-se o trabalho de Ram (1986), o qual afirma que, por meio da elevação da produtividade do setor privado, os gastos públicos conseguem elevar o crescimento econômico, resultado obtido por meio da análise de setores responsáveis pela produção de insumos usados pela iniciativa privada.

De modo diferente, Summers e Heston (1988) estudaram os gastos com defesa e educação e concluíram que esse tipo de despesa é considerado improdutivo. A influência de política fiscal sobre o produto de uma economia também foi discutida por Herrera e Blanco (2006), os quais estimaram esse impacto e concluíram que, em longo prazo, subsídios apresentavam efeitos negativos sobre o produto.

Os gastos públicos e o crescimento também foram explorados em alguns estudos no Brasil, dentre eles, os de Araujo, Monteiro e Cavalcante (2010) e Silva (2012). Este último tratou da relação entre as variáveis, com dados em painel para os municípios paraibanos, entre 
2000 e 2008. O autor concluiu que os gastos com segurança pública, assistência, administração e planejamento não apresentam relação com o produto, enquanto as despesas com habitação e urbanismo apresentaram impacto negativo sobre o produto. Os gastos com o legislativo apresentam relação positiva, e as despesas com educação têm baixa elasticidade-produto, influenciando de forma modesta o crescimento econômico.

Pelo exposto, este trabalho procura analisar os impactos (positivos ou negativos) dos gastos governamentais no crescimento econômico nos municípios do estado do Ceará, tendo em vista que essas variáveis são relevantes à tomada de decisões em políticas públicas, a partir do conhecimento e de uma boa análise interpretativa. As decisões podem contribuir significativamente para uma gestão e alocação eficiente dos recursos públicos.

Dito isso, faz-se necessário conhecer a influência dos gastos públicos nos municípios, através de seu acompanhamento em âmbito municipal, bem como precisar quais trazem retornos positivos aos municípios. Em outras palavras, o objetivo principal deste artigo é verificar quais gastos públicos contribuem para incrementar o produto da economia.

Para atingir o objetivo traçado, utiliza-se o modelo econométrico dinâmico para dados em painel, desenvolvido por Arellano e Bond (1991), Arellano e Bover (1995) e Blundell e Bond (1998), no qual as unidades são os municípios do estado do Ceará, e o período de tempo compreende os anos de 2002 a 2009. A variável dependente a ser estimada será o Produto Interno Bruto (PIB) per capita, que agrega todos os bens e serviços produzidos de um determinado período, em um dado município. As variáveis explicativas serão: os gastos públicos dos governos municipais retirados do relatório elaborado pela Secretaria do Tesouro Nacional STN/FINBRA (2002-2009), o consumo de energia elétrica industrial e comercial, e o número de pessoas com ensino fundamental completo, por município cearense - dados obtidos no Instituto de Pesquisa e Estratégia Econômica do Ceará (IPECE). 
Na sequência, além dessa introdução, o artigo está composto por mais seis seções: a segunda faz uma breve revisão da literatura acerca do tópico em discussão, com ênfase na relação entre crescimento econômico e gasto público. Num terceiro momento, discute-se o modelo de crescimento endógeno de Barros (1990). Na quarta seção, apresenta-se a base de dados. Na quinta, o modelo econométrico será destacado. Na sexta, os resultados são analisados. Na última seção, são apresentadas as considerações finais.

\section{Gastos públicos}

\subsection{0 gasto público e finanças públicas}

Segundo Musgrave e Musgrave (1980), "finanças públicas" é uma terminologia que tem sido tradicionalmente aplicada ao conjunto de problemas da política econômica, envolvendo o uso de medidas de tributação e dispêndios públicos. Nesse caso, investiga-se a existência das falhas de mercado que tornam necessária a presença do governo. Também são analisadas as funções dos governos, a teoria da tributação e do gasto público.

Dentre as falhas de mercados, tem-se a concorrência imperfeita, as externalidades, a informação assimétrica e mercados incompletos, que podem ser corrigidas por algumas políticas públicas, como legislação específica, taxação, entre outras. Por exemplo, uma correção acontece quando o governo controla os preços por meio de tabelamento ou fixação do preço mínimo.

Já os gastos públicos podem ser considerados um dos principais meios de atuação do governo. Por meio deles, o governo manifesta as suas prioridades mediante a prestação de serviços públicos básicos e a realização de investimentos.

Existem vários fatores que afetam o nível dos gastos públicos e suas oscilações: a renda nacional, a capacidade do governo em obter receitas, os problemas sociais, as mudanças políticas, o desenvolvimento tecnológico, os gastos públicos em períodos anteriores 
etc. Desnecessário dizer que eles influenciam no comportamento e no nível de gastos governamentais, direta ou indiretamente, seja aqui no Brasil ou em qualquer país do mundo.

Embora não seja a única forma, pode-se dizer que o tamanho do Estado é mais bem mensurado se medido por meio do gasto público. A outra possibilidade faz referência às diferentes modalidades de financiamento.

Para financiar seus gastos, o governo pode utilizar da emissão de moeda, do lançamento de títulos públicos, dos empréstimos bancários (interno e externo) e da tributação. Cada uma dessas fontes de financiamento provocará consequências diversas sobre as atividades econômicas do país e, independentemente dos efeitos, a tributação tem sido o principal meio de financiamento dos gastos governamentais.

E já que as atividades do governo estão ligadas ao bem-estar dos cidadãos, o contribuinte espera que os gastos que financiam lhes assegurem uma gama de benefícios.

\subsection{Conceitos e classificações}

Conforme Riani (2002), os gastos públicos compreendem a soma de todos os gastos governamentais com a administração (direta e indireta) mais as despesas com atividade econômica produtiva. A sua classificação é realizada conforme a finalidade, natureza e função, desconsiderando os gastos das atividades econômicas do governo. Devido ao grau de detalhamento das informações, a apresentação dos gastos realizados pela administração direta e indireta pode ser dividida em grandes agregados, categorias econômicas ou funções.

De acordo com Rezende (2001), os gastos públicos podem ser classificados de acordo com a finalidade, natureza e agente encarregado da execução do gasto.

Dada certa finalidade, os gastos são classificados em funções, programas, projetos, subfunções etc. Com relação à natureza, os gastos podem ser distribuídos em quatro categorias: custeio, investimento, 
transferências e inversões financeiras. Quanto ao agente encarregado pela execução, podem ser divididos em administração direta e administração indireta.

Essa repartição dos gastos públicos é considerada adequada para examinar a qualidade da despesa realizada por um ente federativo, pois acarreta em informações gerais sobre as operações do governo. Tal classificação tem como objetivo mostrar como são alocados os recursos públicos em diversos setores, tais como: educação, saúde, assistência social, administração, urbanização, saneamento, entre outros.

Salienta-se que todos esses conceitos e classificações são embasados em alguma legislação. Por exemplo, no artigo 12, capitulo II, da Lei 4.320/64, estatuem-se normas gerais de direito financeiro para elaboração e controle dos orçamentos e balanços da União, dos estados, dos municípios e do Distrito Federal. O gasto público é classificado em gasto corrente (despesas de custeio e transferências correntes) e com capital (investimentos, inversões financeiras e transferências de capital) (BRASIL, 1964).

\subsection{Gasto público e crescimento econômico}

O estudo da relação entre gastos públicos e crescimento econômico é discutido há muito tempo. Desde 1820 que Thomas R. Malthus, autor da Lei dos Rendimentos Decrescentes, demonstrara inquietação proveniente dos efeitos da relação entre gasto público e crescimento econômico sobre a economia, como afirma Sant'anna (2006).

Ainda segundo Sant'anna (2006), o economista alemão Adolph Wagner estabeleceu, em 1890, a Lei dos Dispêndios Públicos Crescentes, conhecida também como Lei de Wagner, que tratava do aumento das atividades do Estado. Segundo ele, para que houvesse crescimento da renda per capita, seria necessário que o governo participasse cada vez mais na oferta de bens públicos. Assim sendo, em qualquer estado progressista, seria inevitável que os gastos públicos crescessem mais rapidamente que a renda nacional. Já em 1958, para ele, que foi um dos primeiro a explicar uma relação existente entre gastos públicos e 
crescimento econômico, a expansão das atividades do governo era vista como efeito do progresso social.

Seguem alguns dos motivos utilizados para constatar a tendência do crescimento dos gastos públicos: crescimento populacional, conflitos bélicos, ações para o desenvolvimento e a baixa produtividade do setor público comparada a do setor privado.

Conforme Keynes (1985), existe uma relação simples entre o orçamento do governo e a atividade econômica em seus modelos de demanda agregada, de forma que um corte no déficit do governo reduziria o consumo e o produto. Esse corte seria dado via aumento de impostos e/ou redução dos gastos. É conhecida também uma corrente de linha ortodoxa que se fundamenta na Teoria Ricardiana: a relação entre o crescimento econômico e o gasto público. Nela, o consumidor modifica, de acordo com a política de tributação e gastos do governo, o seu gasto com consumo e poupança.

A discussão existente sobre o tema tem se tornado cada vez mais presente nas preocupações dos pesquisadores. Através desses estudos sobre os gastos do governo e a sua influência sobre o produto, procurase ter uma melhor visão sobre políticas públicas que devam ser tomadas.

\subsubsection{Relação positiva do gasto público com o crescimento econômico}

Nesta seção, são feitas considerações de diversos autores que constataram uma relação positiva entre gastos públicos e o crescimento econômico.

Aschauer (1989), ao analisar a relação dos gastos públicos com o crescimento econômico, foi um dos primeiros a associar a ideia de que, por meio da produtividade do setor privado, os gastos públicos podem vir a elevar o crescimento econômico. Ele utilizou dados da economia dos Estados Unidos da América (EUA) e, ao estimar por Mínimos Quadrados Ordinários (MQO) qual o efeito do gasto público sobre o PIB da economia, obteve que seria possível um aumento de 0,36 a 0,39 no produto, com o 
aumento de 1\% no capital público. Em seu trabalho, buscou explicação para a diminuição nas taxas de crescimento da produtividade nos EUA, que se apresentou na década de 1970. Ademais, obteve resultados significativos da participação do capital público em investimento com infraestrutura sobre o crescimento econômico.

Ram (1986) e Cashin (1995) foram outros autores que analisaram e concluíram que, por meio da elevação da produtividade do setor privado, os gastos públicos elevariam o crescimento econômico, resultado obtido por meio da análise de setores responsáveis pela produção de insumos usados pela iniciativa privada. Para tanto, foram citados serviços de infraestrutura (transporte, telecomunicações e energia) e de defesa nacional, preservando os direitos de propriedade.

No modelo teórico desenvolvido por Barro (1990), o gasto público é incorporado à função de produção para determinar seu efeito sobre o produto da economia. Para ele, a produtividade do setor privado e a acumulação de capital da economia são influenciadas pela estrutura e eficiência na provisão de bens e serviços públicos.

Samuelson e Nordhaus (1990) (apud Sant'anna, 2006) constataram que os níveis de produção e emprego são influenciados de forma significativa pelos gastos públicos, notadamente os de bens e serviços. Os autores sustentaram a importância da eficiência na alocação dos recursos públicos na economia.

Em outro trabalho, Barro (1991) argumentou que os gastos com defesa e educação são considerados produtivos. Isso se daria uma vez que vão proteger os direitos de propriedade através de gastos com segurança. O investimento e o crescimento aumentam, enquanto os gastos com educação deveriam ser compreendidos como investimento em capital humano.

Na mesma perspectiva, Easterly e Rebelo (1993) fizeram uma análise do crescimento econômico entre diferentes países e categorias de investimento público. Para tanto, utilizaram um conjunto de regressões em cross-section e constataram que, para os países em desenvolvimento, 
o investimento público em transporte e comunicação ensejou um notável crescimento econômico.

Devarajan et al. (1996) analisaram 43 países em desenvolvimento ao longo de 20 anos. Constataram que gastos normalmente produtivos, quando feitos em excesso, podem se tornar improdutivos. Sendo assim, observaram que somente se associavam a um maior crescimento econômico os gastos correntes.

Ferreira (1996), Ferreira e Malliagros (1998) e Rocha e Giuberti (2005) seguiram o modelo de Barro (1990): consideraram a quantidade de bens e serviços públicos, capital e trabalho como insumos na função de produção. Esses autores abordaram a influência sobre o crescimento econômico por parte dos gastos públicos agregados, e dos investimentos em infraestrutura no Brasil, e encontraram evidências de relação positiva entre investimentos em infraestrutura (energia, telecomunicações e transportes) e crescimento econômico brasileiro.

O mesmo pensamento é corroborado no trabalho de Cândido Júnior (2001), que analisou a relação entre gastos públicos e crescimento no Brasil durante o período de 1947-1995 e concluiu que, no curto prazo, os gastos públicos defasados no período de um ano possuem impacto positivo sobre o PIB, enquanto no longo prazo esse efeito se reverte.

Santos (2008), ao analisar os municípios do estado do Ceará, concluiu que gastos públicos com capital humano representavam o maior retorno ao PIB per capita, sugerindo uma relação positiva entre maior investimento em educação e crescimento econômico na região. Esse resultado corrobora com os modelos de crescimento econômico de Lucas (1988) e Barro (1990), que apresentam o capital humano como propulsor do crescimento. Santos (2008) constatou ainda que o PIB municipal é pouco sensível, inelástico e influenciado positivamente por gastos com saúde e saneamento.

A pesquisa realizada por Freitas et al. (2009) para o médio prazo no Nordeste brasileiro encontrou resultados significativos tanto para gastos correntes quanto para gastos de capital, sendo o primeiro relacionado 
negativamente e o segundo positivamente com o crescimento econômico. Portanto, a contenção de gastos de custeio e ampliação de investimento público na região foi sugerida para a elevação do produto do Nordeste.

Ademais, existem pesquisadores que não corroboram com a relação positiva entre crescimento econômico e gastos públicos.

\subsubsection{Relação negativa do gasto público com o crescimento econômico}

Para alguns estudiosos do tema, as despesas públicas são consideradas improdutivas e apresentam uma relação negativa com o Produto Interno Bruto. Por exemplo, Bhagwati (1982) e Srinivasan (1985) descrevem a existência de uma relação negativa entre as despesas de consumo do governo e a evolução do produto no longo prazo. Nessas condições, as despesas públicas representam privilégios e interesses de certos grupos

Na mesma perspectiva, Aschauer e Greenwood (1985) defenderam que bens e serviços públicos que entram na função utilidade geram efeitos negativos sobre o PIB, devido à elevação dos impostos para financiálos e, como consequência, uma redução no retorno dos investimentos privados.

Kormendi e Meguire (1985), ao estudarem 47 países, analisaram o crescimento do PIB per capita e a participação do gasto total do governo no PIB, mas não encontraram nenhuma relação significativa. Contudo, ao ampliar o modelo para 115 países, obtiveram uma relação negativa estatisticamente significante entre a participação do governo no PIB e o crescimento real do produto.

Conforme Devarajan et al. (1996), em um estudo ao longo de 20 anos num conjunto de 43 países em desenvolvimento, os gastos com capital, transporte, comunicação, saúde e educação, que são considerados normalmente como produtivos, podem ser improdutivos caso realizados em excesso. 
Schmitz (1996) aponta que, juntamente com a alta arrecadação de receita tributária, os altos gastos públicos nos países em desenvolvimento contribuem para que estes apresentem renda per capita menor que nos países desenvolvidos.

Na mesma linha, Alesina e Perotti $(1995,1997)$ argumentaram serem tangíveis taxas de crescimento mais elevadas, com ajustes fiscais provenientes de cortes nos gastos correntes, em vez de aumentos nos impostos. Seguem o mesmo raciocínio Herrera e Blanco (2006) ao afirmarem que a influência da política fiscal (subsídios) sobre o produto de uma economia acarreta efeitos negativos no longo prazo.

\subsubsection{Relação não significativa do gasto público com o crescimento econômico}

Existem autores que, ao estudarem a relação entre gastos públicos e crescimento econômico, não encontram nenhum tipo de relação (positiva ou negativa) entre essas duas variáveis. Por exemplo, Kormendi e Meguire (1985), ao analisarem 43 países, encontraram resultados não significativos para os efeitos das despesas sobre o produto per capita.

Easterly e Rebelo (1993), ao estimarem regressões em crosssection para países em desenvolvimento e analisarem diferentes categorias de investimentos públicos, não obtiveram evidências estaticamente significativas de que os gastos públicos sejam produtivos.

Para Mazoni (2005), os gastos públicos podem exercer influência de forma direta e indireta sobre o produto da economia, sendo indiretamente por meio dos investimentos privados. Ele afirmou que, no longo prazo, os gastos com consumo do governo e o produto possuem uma relação negativa, todavia, entre os investimentos (públicos e privados) e o produto, essa relação é positiva. Vale salientar que não se apresentou relação entre ambos os investimentos, sendo a influência do investimento privado cerca de 2,6 vezes maior que a do investimento público sobre o PIB. Essa análise foi feita no período de 1970 a 2003 no Brasil. 
No trabalho de Rocha e Giuberti (2005), foi feito um estudo com dados em painel para os estados brasileiros no período de 1986-2002. Elas abordaram os gastos públicos sobre características econômicas (corrente ou de capital) e sobre uma classificação funcional, sendo avaliadas despesas com defesa, educação, saúde, transporte e comunicação. Foi diagnosticada no longo prazo uma relação negativa entre o crescimento econômico e os gastos correntes do governo.

Sant'Anna (2006) analisou a relação entre PIB e gastos sociais dos municípios no estado do Espírito Santo, mas não obteve relação estatística. Em seu trabalho, os gastos sociais municipais estão representados pelos indicadores de assistência e previdência, saúde e saneamento, educação e cultura, habitação e urbanismo.

Finalizando a revisão da literatura acerca da relação entre os gastos públicos e o crescimento econômico, constata-se a existência de controvérsias entre os diversos autores citados, justificando a análise empírica proposta aqui.

\section{Modelo teórico de crescimento endógeno}

O presente artigo baseia-se no modelo de crescimento endógeno desenvolvido por Barro (1990), o qual inclui os gastos do governo financiados por impostos, como insumo na função de produção. Destaca-se a importância dos gastos governamentais sob a forma de investimentos, principalmente em infraestrutura, no processo de crescimento econômico, pois, para ele, existe uma crescente necessidade do governo de intervir diretamente na economia, a fim de gerar externalidades positivas para os produtores e consumidores.

A função de produção do modelo de Barro (1990) é definida da seguinte forma:

$$
y=\Phi(k, g)=k \cdot \Phi\left(\frac{g}{k}\right)
$$

Em que y representa o PIB, k é o estoque de capital privado e $g$ é o gasto do governo, sendo todas essas variáveis em termos per 
capita. Jás $\Phi$ atisfaz as condições de retornos marginais positivos e decrescentes para k e g, ou seja, $\Phi>0$ e $\Phi^{\prime \prime}<0$. Dessa forma, tem-se que o produto marginal do capital na qual implica a função de produção definida em (1) é dado por:

$$
\frac{\partial y}{\partial k}=\Phi(k, \mathrm{~g}) \cdot\left(1-\Phi^{\prime} \cdot \frac{\mathrm{g}}{k}\right)=\Phi(k, \mathrm{~g}) \cdot(1-\eta),
$$

Tal que $\eta$ é a elasticidade de $y$ em relação a $g$ (para um dado valor de $k$ ), assim, $0<\eta<1$. Salienta-se que o produto marginal é determinado pela variação de $k$ na equação (1), mantendo-se $g$ fixo. Assume-se que, para o produtor representativo, as alterações na quantidade de capital e produto não levam a mudanças nos serviços públicos. O autor supõe ainda que os gastos do governo são financiados pela tributação proporcional à renda e que a restrição orçamentária do governo segue expressa da seguinte maneira:

$$
g=T=t y=t k \Phi\left(\frac{g}{k}\right)
$$

Sendo $T$ e $g$, respectivamente, as receitas tributárias e os gastos do governo. A alíquota $t$ compreende a taxa média do imposto de renda e pode ser entendida também como medida de carga tributária incidente sobre a economia.

Segundo Barro (1990), nesse modelo, o agente representativo da economia possui vida infinita e escolhe sua trajetória de consumo maximizando a expressão (4) sujeita às restrições (2) e (3):

$$
U=\int_{0}^{\infty}(u(c)) e^{-\sigma t} \mathrm{dt}
$$

E utilizando a função utilidade dada por:

$$
u(c)=\left(\frac{c^{1-\sigma}-1}{1-\sigma}\right)
$$

Sendo c o consumo per capita e $\sigma>0$, uma taxa constante de preferência intertemporal.

Ademais, resolve-se o modelo hamiltoniano e depois de algumas manipulações algébricas encontra-se a taxa de crescimento econômico da economia expressa por: 


$$
\gamma=\frac{\dot{c}}{c}=\frac{1}{\sigma}\left[(1-\tau) \cdot \Phi\left(\frac{g}{k}\right) \cdot\left(1-\Phi^{\prime} \tau\right)-\rho\right]
$$

Dessa forma, a equação (6) mostra que a taxa de crescimento econômico é função da razão entre os gastos públicos e o capital privado, da carga tributária e do nível tecnológico. Observa-se que a atuação governamental causa dois efeitos ambíguos sobre a taxa de crescimento econômico no longo prazo, a saber: a) os gastos públicos, entrando na função de produção, geram externalidades positivas sobre o capital privado, aumentando a taxa de crescimento da economia; e b) a expansão dos gastos públicos implica necessariamente em um aumento de carga tributária, reduzindo o crescimento econômico, pois diminuiria os recursos disponíveis ao setor privado.

Assim sendo, a política fiscal (gastos públicos e tributação) impacta o crescimento econômico através de duas formas: tem-se um efeito positivo das despesas sobre o PIB e o efeito negativo da tributação, que diminui o retorno líquido do capital privado. Já o efeito total depende do tamanho do governo em termos da relação $(g / k)$ e tributação necessária para financiar as despesas públicas.

Segundo Barro (1990), é possível encontrar um tamanho ótimo de participação do setor público na economia. A relação $g / k$ que maximiza a taxa de crescimento é igual ao seu produto marginal. No entanto, verificase que, caso a participação do governo na economia esteja além do nível ótimo, podem ocorrer diversos impactos sobre a economia, de forma que a expansão dos gastos requer aumento de impostos, diminuindo o retorno dos investimentos. O autor supracitado conclui afirmando que o ponto ótimo depende do grau de eficiência dos gastos públicos.

A partir dessas considerações, e diante da importância de se analisar os impactos dos gastos públicos no crescimento econômico nos municípios do estado do Ceará, a próxima seção discute a base de dados e a seguinte buscará empiricamente estimar as relações entre esses gastos e o crescimento econômico, de forma dinâmica, por meio da metodologia de dados em painel, desenvolvida por Arellano e Bond (1991), Arellano e Bover (1995) e Blundelle Bond (1998). 


\section{Base de dados}

Os dados utilizados na estimação dos modelos econométricos descritos na próxima seção são dos municípios do estado do Ceará para o período de 2002 a $2009^{2}$.

Os gastos públicos dos governos municipais são retirados do relatório elaborado pela Secretaria do Tesouro Nacional - STN/FINBRA (2002-2009), que apresenta informações sobre a execução orçamentária (receita; despesa orçamentária; despesas por função e subfunção) e demonstrativos patrimoniais dos municípios no Brasil.

Desses relatórios, retiraram-se especificamente as seguintes despesas: saúde e saneamento (DSS), urbanismo e habitação (DUR), legislativo (DL) e custeio com pessoal (DP). Essas variáveis foram transformadas em termos per capita, dividindo-se o valor pela população do município correspondente. A análise apresentada na próxima seção irá confirmar quais dessas despesas contribuem no incremento ou diminuição do crescimento econômico municipal cearense. Portanto, os sinais dos parâmetros estimados no modelo econométrico devem ser positivos ou negativos, conforme discussão na seção 2 .

Foi obtido no Instituto de Pesquisa e Estratégia Econômica do Ceará (IPECE) o consumo de energia elétrica industrial e comercial (CF) para ser utilizada como proxy do estoque de capital físico e o número de pessoas com ensino fundamental completo a ser usada como proxy do capital humano (CH), por município cearense, de 2002 a 2009. Conforme exposto na seção 2, espera-se que essas variáveis contribuam para elevar o produto dos municípios. Ressalta-se que elas geralmente são utilizadas como proxies em trabalhos desse tema, conforme Araujo, Monteiro e Cavalcante (2010), Silva (2012), entre outros (IPECE, 2011).

Considerou-se 122 municípios sem função da não disponibilidade de dados para todos os 184 do estado do Ceará no período em análise. 
As informações sobre o PIB per capita de cada município foram obtidas no Instituto de Pesquisa Econômica Aplicada (IPEA, 2011).

Ressalta-se que todas as variáveis monetárias foram atualizadas para valores reais de 2009 utilizando o Índice Nacional de Preços ao Consumidor (INPC), tendo como base o ano de 2009 (IPEA, 2011).

\section{Modelo econométrico}

Com o intuito de verificar o impacto dos gastos públicos municipais sobre o PIB per capita dos municípios cearenses, utilizam-se os estimadores do Método de Momentos Generalizado (MMG-sistema), desenvolvido nos trabalhos de Arellano e Bond (1991), Arellano e Bover (1995) e Blundelle Bond (1998). As relações econômicas, muitas vezes, são de natureza dinâmica, definidas a partir da interação contínua dos seus mecanismos internos. Essa proposição geral é certamente válida quando se analisa o processo de crescimento econômico - no presente trabalho, o PIB per capita.

Na esfera da análise empírica, a metodologia de dados em painel possibilita o melhor entendimento do caráter dinâmico. Assim sendo, a relação entre o PIB per capita e os gastos públicos determinantes são investigados por meio do seguinte modelo de regressão para dados em painel:

$$
\begin{gathered}
\ln \left[P I B_{i t}\right]=\beta_{0}+\beta_{1} \ln \left[P I B_{i t-1}\right]+\beta_{2} \ln \left[C F_{i t}\right]+\beta_{3} \ln \left[C H_{i t}\right]+\beta_{4} \ln \left[D P_{i t}\right]+ \\
\beta_{5} \ln \left[D S S_{i t}\right]+\beta_{6} \ln \left[D L_{i t}\right]+\beta_{7} \ln [D U R]+\eta_{i}+\varepsilon_{i t}
\end{gathered}
$$

Em que a variável coeficiente de $P I B_{i t}$ é Produto Interno Bruto per capita; $P I B_{i t-1}$ é o Produto Interno Bruto per capita defasado; $C F_{i t}$ é a medida de capital físico (energia elétrica) per capita; $\mathrm{CH}_{i t}$ é a medida de Capital Humano representada pelo número de indivíduos que concluíram o ensino fundamental; $D P_{i t}$ é a despesa de custeio com pessoal per capita; $D_{S S}$ é a despesa com saúde e saneamento per capita; $D L_{i t}$ é despesa com o legislativo per capita; $D U R_{i t}$ é a despesa com urbanismo e habitação; $\eta_{i}$ são os efeitos aleatórios não observáveis e $\varepsilon_{i t}$ representa os distúrbios aleatórios. As variáveis do modelo (7) são definidas em 
logaritmo natural, em que o subscrito $i$ representa o município e $t$, o período de tempo.

Conforme Ahn e Schmidt (1995), esse modelo possui as seguintes hipóteses: $\mathrm{E}\left[\eta_{i}\right]=\mathrm{E}\left[\eta_{i} \varepsilon_{t}\right]=0$ e $\mathrm{E}\left[\eta_{i t} \varepsilon_{t s}\right]=0$ para $i=1,2, \ldots ., N$ e $\forall \mathrm{t} \neq \mathrm{S}$. Há também a hipótese padrão relativa às condições iniciais: $P I B_{i t-1}: E\left[P I B_{i t-1}\right.$ $\left.\varepsilon_{i t}\right]=0$ para $i=1,2, \ldots ., N$ e $t=1,2, \ldots . ., T$.

O trabalho de Arellano e Bond (1991) destaca que existem problemas econométricos ao estimar o modelo (7) por meio de técnicas de estimação tradicionais (mínimos quadrados ordinários). Devido à presença dos efeitos não observáveis dos indivíduos, $\eta_{i}$, juntamente com a variável dependente defasada, $P I B_{i t-1}$, no lado direito da equação (7). Nesse caso, omitir os efeitos fixos individuais no modelo dinâmico em painel torna os estimadores de MQO enviesados e inconsistentes.

A presença da variável $P I B_{i t-1}$ como variável explicativa no modelo (7) resulta, em geral, num problema de endogeneidade com o termo de efeitos fixos, o que provoca um viés no painel dinâmico. Assim sendo, a estimação do modelo (7) por MQO produz estimadores inconsistentes dos parâmetros, além de superestimar o coeficiente de $P I B_{i t-1}$. O mesmo problema pode se reproduzir em qualquer outra variável explicativa do modelo.

Uma maneira de superar esse problema seria eliminar a presença dos efeitos fixos no modelo (7). Assim, uma primeira tentativa seria estimar o modelo (7) por meio de MQO com variáveis dummies para cada município do estado ou através do método WITHIN GROUPS, que gera as mesmas estimativas do método anterior, mas com os desvios padrões dos coeficientes ligeiramente menores. Os estimadores do coeficiente de $P I B_{i t-1}$ por ambos os métodos serão menores do que o obtido por MQO. De qualquer maneira, pode-se mostrar que o viés no painel dinâmico ainda continua a existir.

A lição que se tira é que uma "boa" estimativa do parâmetro de $P I B_{i t-1}$ deve estar compreendida entre os limites dos estimadores obtidos por MQO e WITHIN GROUPS. Nesse sentido, faz-se necessária alguma transformação no modelo (7) que expurgue os efeitos fixos, eliminando 
definitivamente o problema da endogeneidade. Para solucionar esses problemas, Arellano e Bond (1991) propuseram o estimador do método dos momentos generalizado-diferenciado (MMG-diferenciado). Tal método consiste na eliminação dos efeitos fixos através da primeira diferença da equação (7), da seguinte forma:

$$
\begin{gathered}
E\left[\eta_{i}\right]=E\left[\varepsilon_{i t}\right]=E\left[\eta_{i} \varepsilon_{i t}\right]=0 \text { e } E\left[\varepsilon_{i t} \varepsilon_{i s}\right]=0 \text { para } i=1,2, \ldots ., N \text { e } \\
P I B_{i t-1}: E\left[P I B_{i t-1} \varepsilon_{i t}\right]=0 \text { para } i=1,2, \ldots, N \text { e } t=1,2, \ldots \ldots, T .
\end{gathered}
$$

Tal que, para qualquer variável, In $y_{i t}, \Delta \ln y_{i t}=\ln y_{i t}-\ln y_{i t-1}$. Note que, na equação (8), $\Delta \ln P I B_{i t-1}$ e $\Delta$ In $\varepsilon_{i t}$ são correlacionados e, assim sendo, estimadores de MQO para seus coeficientes serão enviesados e inconsistentes. Portanto, faz-se necessário utilizar variáveis instrumentais para $\triangle \ln P I B_{i t-1}$.

As hipóteses adotadas na equação (8) implicam que as condições de momentos $\mathrm{E}\left[\Delta \ln P I B_{i t-s} \Delta \ln \varepsilon_{i t}\right]=0$, para $t=3,4, \ldots . T$ e $s \geq 2$, são válidas. Baseados nesses momentos, Arellano e Bond (1991) sugerem empregar In $P I B_{i t-s}$, para $\mathrm{t}=3,4, \ldots . . \mathrm{T}$ e $\mathrm{s} \geq 2$, como instrumento para equação (8).

As demais variáveis explicativas podem ser classificadas como: (a) estritamente exógena, se não é correlacionada com os termos de erro passados, presente e futuros; (b) fracamente exógena, se é correlacionada apenas com valores passados do termo de erro; e (c) endógena, se é correlacionada com os termos de erro passado, presente e futuro. No segundo caso, os valores da variável defasada em um ou mais períodos são instrumentos válidos na estimação da equação (8) e, no último caso, os valores defasados em dois ou mais períodos são instrumentos válidos na estimação dessa equação.

Conforme Arellano e Bover (1995) e Blundell e Bond (1998), esses instrumentos são fracos quando as variáveis dependentes e explicativas apresentam forte persistência e/ou a variância relativa dos efeitos fixos aumenta. Isso produz um estimador MMG-diferenciado não consistente e enviesado para painéis com $T$ pequeno.

Assim sendo, Arellano e Bover (1995) e Blundell e Bond (1998) propuseram um sistema que combina o conjunto de equações em 
diferença - equação (8) - com o conjunto de equações em nível - equação (7) - para reduzir esse problema de viés. Esse sistema é denominado "método dos momentos generalizado-sistema" (MMG-sistema). Para as equações em diferenças, o conjunto de instrumentos é o mesmo descrito anteriormente. Para regressão em nível, os instrumentos apropriados são as diferenças defasadas das respectivas variáveis.

As estimativas do MMG-sistema apresentadas na próxima seção resultam da estimação com estimador corrigido pelo método de Windmeijer (2005), para evitar que o respectivo estimador das variâncias subestime as verdadeiras variâncias em amostra finita. O estimador utilizado foi proposto por Arellano e Bond (1991) em dois passos. Na primeira etapa, supõe-se que os termos de erro são independentes e homocedásticos nos estados e ao longo do tempo. No segundo estágio, os resíduos obtidos na primeira etapa são utilizados para construir uma estimativa consistente da matriz de variância-covariância, relaxando, assim, as hipóteses de independência e homocedasticidade. O estimador do segundo estágio é assintoticamente mais eficiente em relação ao estimador da primeira etapa.

A consistência do estimador MMG-sistema depende da suposição de ausência de correlação serial no termo de erro e da validade dos instrumentos adicionais. Assim sendo, inicialmente, testam-se as hipóteses nulas de ausência de autocorrelação de primeira e segunda ordem dos resíduos. Para que os estimadores dos parâmetros sejam consistentes, a hipótese de ausência de autocorrelação de primeira ordem deve ser rejeitada e a de segunda ordem, aceita. Posteriormente, realiza-se o teste de Hansen e Sarganpara para verificar a validade dos instrumentos utilizados.

\section{Resultados e discussões}

Esta seção apresenta e discute os resultados obtidos da estimação do modelo econométrico apresentado na seção anterior. Os resultados estimados do modelo (7) por MQO, WITHIN GROUPS e MMG-sistema para o PIB per capita se encontram dispostos na Tabela 1. 
Foram usadas como variáveis endógenas a variável dependente $P I B_{i t-1}$ defasada de um período e as despesas legislativas per capita. As demais variáveis explicativas foram consideradas fracamente exógenas. Toda a equação estimada é logaritimizada, a fim de se obter as elasticidades por meio dos coeficientes. Todas as variáveis estimadas apresentam significância esperada.

Os testes efetuados no modelo MMG-sistema revelam que as propriedades estatísticas do modelo são aceitáveis. Os testes de Hansen e Sargan testam, respectivamente, se os instrumentos utilizados e os instrumentos adicionais requerido pelo MMG-sistema são válidos. Por último, incluem-se os testes estatísticos de Arellano e Bond (1991), para avaliar a existência de autocorrelação de primeira e segunda ordem. Note que a ausência de autocorrelação de segunda ordem é essencial para a consistência do estimador MMG-sistema. O teste confirma a não rejeição de autocorrelação de primeira ordem, embora se rejeite a hipótese de autocorrelação de segunda ordem.

Observa-se que na coluna [a] na Tabela 1 os valores dos coeficientes estimados da variável In PIB ${ }_{i t-1}$ por MQO são de fato maiores do que os valores estimados na coluna [b] para essa mesma variável por WITHIN GROUPS. Assim, se os instrumentos utilizados forem adequados, os valores dos coeficientes dessa variável estimados por MMG-sistema devem ficar situados entre os limites dos coeficientes estimados pelos dois métodos anteriores.

Os valores obtidos por MMG-sistema para essa variável na coluna [c] mostra que essa característica é satisfeita. Isso indica que o viés causado pela presença de variáveis endógenas no lado direito da regressão e efeitos fixos não observáveis foram corrigidos por MMG-sistema. Assim sendo, discute-se aqui somente os coeficientes estimados do modelo MMG-sistema dispostos na coluna [c] da Tabela 1.

Observa-se que os coeficientes das variáveis $\mathrm{PIB}_{\mathrm{t}-1}, \mathrm{CF}_{\mathrm{it}} \mathrm{CH}_{\mathrm{it}}, \mathrm{DP}_{\mathrm{it}}$, DUR $_{\text {it }}$ apresentam sinais positivos, portanto, o aumento nos valores dessas variáveis provocam incrementos no PIB per capita dos municípios cearenses. 
Tabela 1 : Resultados dos Modelos de Regressão para InPIB

\begin{tabular}{|c|c|c|c|c|c|c|}
\hline \multicolumn{3}{|c|}{$\begin{array}{l}\mathrm{MQO} \\
{[\mathrm{a}]}\end{array}$} & \multicolumn{2}{|c|}{$\begin{array}{c}\text { WITHIN GROUPS } \\
{[\mathrm{b}]}\end{array}$} & \multicolumn{2}{|c|}{$\begin{array}{l}\text { MMG -Sistema } \\
\text { [c] }\end{array}$} \\
\hline & Coefic. & Valor-p & Coefic. & Valor-p & Coefic. & Valor-p \\
\hline $\mathrm{PIB}_{\mathrm{it}-1}$ & $\begin{array}{c}0,9239 \\
(0,0195)\end{array}$ & 0,00 & $\begin{array}{c}0,4034 \\
(0,0311)\end{array}$ & 0,00 & $\begin{array}{c}0,4904 \\
(0,1446)\end{array}$ & 0,04 \\
\hline \multirow{2}{*}{$C F_{i t}$} & 0,0427 & \multirow{2}{*}{0,01} & 0,0196 & \multirow{2}{*}{0,00} & 0,0591 & \multirow{2}{*}{0,00} \\
\hline & $(0,0017)$ & & $(0,0056)$ & & $(0,0165)$ & \\
\hline \multirow{2}{*}{$\mathrm{CH}_{i t}$} & 0,02657 & \multirow{2}{*}{0,04} & 0,0219 & \multirow{2}{*}{0,32} & 0,1432 & \multirow{2}{*}{0,00} \\
\hline & $(0,0130)$ & & $(0,0222)$ & & $(0,0498)$ & \\
\hline \multirow{2}{*}{$D P_{i t}$} & 0,0243 & \multirow{2}{*}{0,00} & 0,0372 & \multirow{2}{*}{0,00} & 0,0512 & \multirow{2}{*}{0,06} \\
\hline & $(0,0068)$ & & $(0,0065)$ & & $(0,0274)$ & \\
\hline \multirow{2}{*}{$D S S_{i t}$} & 0,0105 & \multirow{2}{*}{0,13} & 0.0273 & \multirow{2}{*}{0,00} & 0,0241 & \multirow{2}{*}{0,44} \\
\hline & $(0,0070)$ & & $(0,0077)$ & & $(0,3133)$ & \\
\hline \multirow{2}{*}{$D L_{i t}$} & $-0,0139$ & \multirow{2}{*}{0,12} & 0,0674 & \multirow{2}{*}{0,00} & $-0,0047$ & \multirow{2}{*}{0,92} \\
\hline & $(0,0090)$ & & $(0,0135)$ & & $(0,0483)$ & \\
\hline$D U R_{i t}$ & $\begin{array}{c}0,0078 \\
(0,0040) \\
\end{array}$ & 0,05 & $\begin{array}{c}0,0118 \\
(0,0049) \\
\end{array}$ & 0,01 & $\begin{array}{c}0,0284 \\
(0,0103) \\
\end{array}$ & 0,00 \\
\hline \multirow{2}{*}{ Const. } & $-0,0069$ & \multirow{2}{*}{0,90} & $-0,1151$ & \multirow{2}{*}{0,26} & 0,6245 & \multirow{2}{*}{0,01} \\
\hline & $(0,0607)$ & & $(0,1030)$ & & $(0,248)$ & \\
\hline \multicolumn{3}{|c|}{$\begin{array}{c}F(7,846)=1792,69 \\
\text { Prob }>F=0,0000 \\
R^{2}=0,93\end{array}$} & \multicolumn{2}{|c|}{$\begin{array}{c}F(7,725)=142,68 \\
\text { Prob }>F=0,0000\end{array}$} & \multicolumn{2}{|c|}{$\begin{array}{l}F(6,121)=64,05 \\
\text { Prob }>F=0,0000\end{array}$} \\
\hline \multicolumn{3}{|c|}{$\mathrm{N}^{\circ}$ de obs: 854} & \multicolumn{2}{|c|}{$\begin{array}{l}N^{0} \text { de obs: } 854 \\
N^{0} \text { de grupos: } 122\end{array}$} & \multicolumn{2}{|c|}{$\begin{array}{l}\mathrm{N}^{\circ} \text { de obs: } 854 \\
\mathrm{~N}^{\circ} \text { de grupos: } 122 \\
\mathrm{~N}^{0} \text { de instrum.: } 11\end{array}$} \\
\hline \multicolumn{3}{|c|}{$\begin{array}{l}\mathrm{H}_{0}: \quad \text { Ausência } \quad \text { de } \\
\text { Autocorrelação nos resíduos } \\
\text { de primeira ordem }\end{array}$} & \multicolumn{2}{|c|}{ Valor-p } & & \\
\hline $\begin{array}{r}\mathrm{H} \\
\text { Autocor } \\
\mathrm{de}\end{array}$ & $\begin{array}{l}\text { Ausência } \\
\text { elação nos } \\
\text { egunda or }\end{array}$ & $\begin{array}{l}\text { e } \\
\text { esíduos } \\
\text { em }\end{array}$ & Valo & & & \\
\hline & te de Hans & & Prob> & & & \\
\hline & te de Sarg & & Prob> & & & \\
\hline
\end{tabular}

Obs.: (i) Os valores em parênteses são os desvios padrões corrigidos pelo método de Windmeijer (2005); (ii) Os valores para o teste de Hansen são os valores-p para a hipótese nula de que os instrumentos são válidos. (iii) Os valores para o teste de Sargan são os valores-p para validade dos instrumentos adicionais requeridos pelo método-sistema.

Fonte: resultados obtidos pelo autor (2012) 
Verifica-se que a hipótese da natureza dinâmica das relações econômicas nos municípios cearenses foi comprovada pelo resultado significativo da variável explicativa "Produto Interno Bruto defasado de um período $\mathrm{PIB}_{\mathrm{t}-1}$ " na coluna [c] da Tabela 1. Em termos de elasticidade, um aumento de $1 \%$ no produto dessa variável (coeterisparibus) ocasiona um incremento de $0,29 \%$ no produto per capita do período seguinte, corroborando com Araujo, Monteiro e Cavalcante (2010) e Candido Jr (2001).

A variável "despesa com capital físico $\mathrm{CF}_{\text {it }}$ apresentou coeficiente significativo com sinal esperado. A função estimada sugere que incrementos do capital físico produzem aumento do crescimento econômico nos municípios cearenses, tendo em vista a contribuição dessa variável na melhoria das condições de vida da população residente no Estado do Ceará. Em termos de elasticidade, um aumento de 1\% dessa variável corresponderia ao incremento de $0,05 \%$ no crescimento econômico dos municípios cearenses. Deve ser ressaltado que, apesar dessa relação entre capital físico e crescimento econômico ser positiva, o indicador possui baixa elasticidade, ou seja, a reação que essa variável provoca no crescimento econômico é modesta.

Já um aumento de $1 \%$ no capital humano corresponderia a um aumento de $0,14 \%$ do PIB per capita, ou seja, um aumento dessa variável contribui para incrementos no crescimento do PIB, corroborando com trabalhos na literatura. Sugere-se, portanto, que políticas públicas que proporcionem incrementos de capital humano sejam efetivas, para dinamizar a economia e gerar crescimento econômico. Esses resultados corroboram com Lucas (1998) e Devarajan et al. (2006), ao afirmarem que o capital humano configura-se como uma importante variável para o crescimento econômico.

Assim sendo, verifica-se que o impacto do capital humano, no nível do produto dos municípios cearenses, é superior ao do capital físico, corroborando com os resultados de Silva (2012), que investigou os impactos dos gastos públicos nos municípios paraibanos.

Dessa forma, pode-se sugerir que os governantes devem proporcionar aumento do capital físico e humano, pois o aprimoramento 
da infraestrutura física, bem como do estoque de capital humano dos municípios cearenses, dinamizaria a economia, por meio do incremento em produtividade, acréscimos na renda e aumento do emprego, o que acarretaria maior crescimento econômico.

As despesas com urbanismo e habitação apresentaram coeficiente significativo e positivo, ou seja, um aumento de 1\% dessas despesas acarretaria uma elevação de aproximadamente 0,03\% do PIB per capita. Já as despesas com pessoal apresentam sinal positivo e significativo. Verifica-se que um aumento de $1 \%$ dessas despesas acarretaria um acréscimo de $0,05 \%$ do PIB per capita, corroborando com os resultados apresentados em nível municipal por Araujo, Monteiro e Cavalcante (2010), os quais analisaram o impacto dos gastos públicos sobre o crescimento econômico dos municípios cearenses no período 2005-2008.

As variáveis "despesas com saúde e saneamento" $\left(D S_{i t}\right)$ e a variável "despesa com o legislativo" $\left(\mathrm{DL}_{\mathrm{it}}\right)$ não apresentaram coeficientes significativos. Comparando-se com alguns resultados encontrados por Santos (2008), em sua análise também para municípios cearenses, observou-se resultado divergente quanto às despesas com saúde e saneamento, visto que se apresentaram em uma relação positiva com o PIP per capita. Em seu trabalho, também destaca que o capital físico como inelástico e o capital humano com resultado significativo e bem elevado sobre o crescimento econômico nos municípios cearenses.

\section{Considerações finais}

Este artigo analisou a dinâmica dos gastos públicos e seus impactos no crescimento econômico nos municípios cearenses empregando metodologia de dados em painel. Em primeiro lugar, os resultados mostraram que as despesas com saúde e saneamento e gastos com o legislativo não têm impacto significativo no crescimento econômico do estado do Ceará, não corroborando, assim, com a hipótese daqueles que afirmam que essas despesas estimulam significativamente 0 crescimento. 
Com relação aos outros determinantes, o crescimento do produto percapita e o incremento de capital físico e capital humano têm contribuído para elevar o crescimento nos municípios cearenses. Ressalte-se que o impacto do capital humano é maior do que o obtido via crescimento do capital físico per capita. Observou-se que para cada aumento de $1 \%$ em capital humano o produto eleva-se em aproximadamente 0,14\%, enquanto para cada aumento de $1 \%$ do capital físico per capita o crescimento do produto é somente de $0,05 \%$. Nesse sentido, políticas públicas direcionadas ao aumento da educação apresentam maiores impactos no aumento do produto do que aquelas que influenciam apenas o crescimento do produto por incrementos de capital físico.

Assim sendo, é imprescindível a orientação e formulação de políticas públicas focadas na educação, elemento que pode ser visto com extrema importância para aumentos de produtividade e determinantes de crescimento de longo prazo nos municípios do Ceará.

Por outro lado, as despesas públicas com pessoal per capita influenciam de forma positiva o aumento do PIB na região durante o período analisado. Cada aumento de 1\% dessa despesa acarretaria um acréscimo de $0,03 \%$ do PIB per capita. Na mesma perspectiva, um aumento de $1 \%$ em urbanismo eleva aproximadamente $0,03 \%$ do PIB per capita. Isso mostra a importância de políticas públicas direcionadas a aumentar despesas com pessoal e urbanismos dos municípios, o que contribui para incrementos do crescimento econômico.

Por fim, propõe-se em trabalhos futuros uma investigação em cada município relacionando o PIB com outros gastos públicos, considerando as fontes de financiamento, objetivando auxiliar a formulação de políticas públicas municipais.

\section{Referências}

ARAUJO, J. A.; MONTEIRO, V. B.; CAVALCANTE, C. A. A influência dos gastos públicos no crescimento econômico dos Municípios do Ceará. In: CARVALHO, E.B.S (org). Encontro economia do Ceará em debate 2010. Fortaleza: IPECE, 2010.p.176-200. 
AHN, S. C.; SCHIMDT, P. Efficient estimation of models for dynamic panel data. Journal of Econometrics. New Jersey, v. 68, p. 5-27, jan, 1995.

ALESINA, A.; PEROTTI, R. Fiscal adjustments in OECD countries: composition and macroeconomic effects. IMF staff papers, New York, v. 44, n. 2, p.210-248, jun.1997.

ALESINA, A.; PEROTTI, R.Fiscal expansions and fiscal adjustments in OECD countries. Economic Policy, Paris, v. 21, p. 207-247, ago, 1995.

ARELLANO, M.; BOVER, O. Another look at the instrumental-variable estimation of error-components model. Journal of econometrics. New York, v. 68, p. 29-52, ago, 1995.

ARELLANO, M.; BOVER, O. ; BOND, S. Some tests of specification for panel data: Monte Carlo evidence and na application to employment equations. The review of economic studies, Stockholm, v. 58, n. 2, p.277-297, abr, 1991.

ASCHAUER, D. Is public expenditure productive? Journal of monetary economics, Chigaco, v. 23, p.177-200, abr, 1989.

ASCHAUER, D. ; GREENWOOD, J. Macroeconomic effects of fiscal policy. Carnegie-Rochester series on public policy, Rochester, v.23, p.91-138, dez, 1985.

BARRO, R.J. Economic growth in a cross-section of countries. The quarterly journal of economics, Cambridge, v.106, p.407-444, nov, 1991.

BARRO, R.J. Government spending in a simple model of endogenous growth. The journal of political economy, Chigaco, v.98, n.5, p.103125, ago, 1990.

BHAGWATI, J. Directly Unproductive Profit-Seeking DUP Activities. Journal of political economy, Chigaco, v. 90, n. 5, p.988-1002, set, 1982. 
BLUNDELL, R.; BOND, S. Initial conditions and moment restrictions in dynamic panel data models. Journal of econometrics, New York, v. 87, p. 115-143, nov, 1998.

BRASIL. Ministério de Orçamento e Gestão. Lei n 4.320, de 17 de março de 1964. Diário Oficial da República Federativa do Brasil. Brasília, 17 mar. 1964. Disponível em: <http://www.stn.fazenda.gov.br/ legislacao/leg_contabilidade.asp>. Acesso em: 05 jan. 2011.

CASHIN, P. Government Spending, Taxes and Economic Growth. IMF Staff Papers, New York, v. 42, n. 2, p. 237-269, set, 1995.

CÂNDIDO JUNIOR, J. O. Os gastos públicos no brasil são produtivos?. Brasília: IPEA, 2001. (Texto para Discussão N781).

DEVARAJAN, S.; SWARROP, V.; ZOU, H. The composition of public expenditure and economic growth. Journal of monetary economics, Chicago, v. 37, p. 313-344, ago, 1996.

EASTERLY, W.; REBELO, S. Fiscal policy and economic growth: an empirical investigation. Journal of monetary economics, Chicago, v. 32, p. 417-458, fev, 1993.

FERREIRA, P. C. Investimento em infra-estrutura no Brasil: fatos estilizados e relações de longo prazo. Pesquisa e planejamento econômico, Brasília, v. 26, n. 2, p. 231-252, jun,1996.

FERREIRA, P. C. ; MALLIAGROS, T.G. Impactos produtivos da infraestrutura no Brasil 1950/95. Pesquisa e planejamento econômico, Brasília, v.28 n.2, p. 315-338, abr, 1998.

FREITAS, U. R. P.; CASTRO NETO, A. A. de; LÔU, I. C. Relação entre Gastos Públicos e Crescimento Econômico: uma análise com dados em painel para o Nordeste. Revista Conj. \& Planej., Salvador, n. 162, p. 50-57, jan./mar. 2009.

HERRERA, S.; BLANCO, F. The quality of fiscal adjustment and the longrun growth impact of fiscal policy in Brazil, In: ENCONTRO NACIONAL 
DE ECONOMIA, 34, 5 a 8 de dezembro de 2006, Salvador. Anais... Salvador: Anpec, 2006.p. 12-32.

IPECE. Instituto de Pesquisa e Estratégia Econômica do Ceará. Ceará em números. Disponível em:<http://www.ipece.gov.br>. Acesso em: 15 jan. 2011.

IPEA. Instituto de Pesquisa Econômica Aplicada. Ipeadata. Disponível em: <http://www.ipeadata.gov.br>.Acesso em: 10 jan. 2011.

KEYNES, J. M. Teoria geral do emprego, do juro e da moeda. São Paulo: Nova Cultural, 1985.

KORMENDI, R.C.; MEGUIRE, P.G. Macroeconomic determinants of growth. Journal of monetary econometrics, Chicago, v. 16, p. 141163, nov, 1985.

LUCAS, R. E. On the mechanics of economic development. Journal of monetary economics, Chicago, v. 22, p. 3-42, dez,1988.

MAZONI, M. G. Gastos públicos e crescimento econômico no Brasil: análise dos impactos dos gastos com custeio e investimento. 2005. 115f. Dissertação (Mestrado em Economia). Faculdade de Economia, Administração e Contabilidade da Universidade de São Paulo (FEAUSP), São Paulo, 2005.

MUSGRAVE, R.; MUSGRAVE, P. Finanças públicas: teoria e prática. São Paulo: Campus/EDUSP, 1980.

RAM, R. Government size and economic growth: a new framework and some evidence from cross-section and time-series data. The american economic review. Virgínia, v. 76, n. 1, p. 191-203, jan, 1986.

REZENDE, F. Finanças públicas. 2 ed. São Paulo: Atlas, 2001.

RIANI, F. Economia do setor público: uma abordagem introdutória. 4 ed. São Paulo: Atlas, 2002.

ROCHA, F.; GIUBERTI, A. C. Composição do gasto público e crescimento econômico: um estudo em painel para os Estados brasileiros. In: 
ENCONTRO NACIONAL DE ECONOMIA, 33, 6 a 9 de dezembro de 2005, Natal, 2005. Anais... Natal: ANPEC, 2005. p. 80-112.

SANT'ANNA, J. M. B. Efeito do gasto público sobre o PIB: um teste empírico nos Municípios do Estado do Espírito Santo. 2006. $101 \mathrm{f}$. Dissertação (Mestrado em Ciências Contábeis). FUCAPE. Vitória, 2006. SANTOS, R. C. L.O efeito do gasto público sobre o PIB dos municípios cearenses. 2008. 80f. Dissertação (Mestrado em Economia). Universidade Federal do Ceará (UFC). Fortaleza, 2008.

BRASIL. SECRETARIA DO TESOURO NACIONAL. STN/FINBRA (2002-2009). Base de dados. Brasília, 2011, Ministério da Fazenda, Brasília, v.1, n.1, 80p, 2011. Disponível em: <http://www.tesouro. fazenda.gov.br>. Acesso em: 10 ago 2011.

SCHMITZ, J. The Role played by public enterprises: how much does it differ across countries. Federal Reserve Bank of Minneapolis Quarterly Review. New York, v. 20,n. 2, p. 2-15, jul. 1996.

SOLOW, R. M. A Contribution to the Theory of Economic Growth. Quarterly Journal of Economics. Cambridge, v. 70, n. 1 p. 65-94, jan,1956.

SRINIVASAN, T. Neoclassical Political Economy, the State, and Economic Development. Asian development review. Mandaluyong, v. 3, n. 2, p. 38-58, abr, 1985.

SILVA, L. M.C. A relação entre os gastos públicos e o crescimento econômico: uma análise para os municípios paraibanos no período 2000 - 2008. 2012. 101f. Dissertação (Mestrado em Economia) Universidade Federal da Paraíba (UFPB). João Pessoa, 2012.

SUMMERS, R.; HESTON, A. A new set of international comparisons of real product and price levels. Estimates for 130 countries. Review of income and wealth, Boston, v. 34, p. 1-25, jan, 1988.

WAGNER, Adolph. Three extracts on public finance. In: MUSGRAVE, R.A.; PEACOCK, A.T. Classics in the theory of public finance. Londres: Macmillan, 1958. p. 32-55. 
WINDMEIJER, F. A finite sample correction for the variance of linear efficient two-step GMM estimators. Journal of econometrics, New Jersey, v. 126, p. 25-51, out, 2005.

Artigo recebido em: 19/09/2013

Aprovado em: 23/04/2014 